\title{
Fünf Tage fokussieren - Zusatzausbildung „Journalismus und Recht“
}

\author{
Katharina König \\ Beisitzerin im Bundesvorstand, Wiss. Mitarbeiterin, Freiburg i.Br.
}

Nicht Neugier, fokussieren sei die wichtigste Journalistentugend. Mit einem launigen Artikel von Harald Martenstein, Journalist beim Berliner Tagesspiegel, begann Karin Völker, Redakteurin der Westfälischen Nachrichten, im Rahmen der Zusatzausbildung „Journalismus und Recht“ der Westfälischen Wilhelms-Universität Münster ihren Vortrag. Tatsächlich stellte sich während des fünftägigen Ausbildungsgangs schnell heraus, dass darin auch eine große Kunst besteht.

Prof. Dr. Thomas Hoeren vom Institut für Informations-, Telekommunikations- und Medienrecht bietet diese Zusatzausbildung nun schon seit fast zehn Jahren an. Hier haben Juristinnen und Juristen aller Altersgruppen eine deutschlandweit einmalige Gelegenheit, ihren Traum vom Journalismus zu überprüfen. Um an dem Seminar teilzunehmen, muss man nicht an der Universität Münster immatrikuliert sein. Bewerben können sich Juristinnen und Juristen aus ganz Deutschland. Neben den üblichen Bewerbungsunterlagen wird eine Arbeitsprobe verlangt. Auf die etwa 15 Plätze bewerben sich jährlich zwischen 100 und 150 Interessierte.

Das Programm der Zusatzausbildung ist umfangreich und anspruchsvoll, für Sightseeing bleibt keine Zeit. Die Teilnehmerinnen und Teilnehmer werden in die Grundtechniken journalistischen Arbeitens eingeführt und bekommen einen Einblick in den Arbeitsalltag von Journalistinnen und Journalisten. Die routinierten Referentinnen und Referenten bilden ein breites Spektrum von Tätigkeitsbereichen im Journalismus ab. Vertreten sind klassische Berufsfelder wie die Arbeit bei TV und Printmedien, aber auch das vergleichsweise junge Berufsfeld des Pressesprechers/der Pressesprecherin einer Großkanzlei oder die Arbeit im Online-Journalismus.

„Die Gemeinsamkeit von Juristerei und Journalismus ist das Handwerkszeug: die Sprache. Juristen wie Journalisten wollen verstanden werden “, stellt Prof. Dr. Joachim Jahn von der Frankfurter Allgemeinen Zeitung fest. Den besonderen Reiz der Zusatzausbildung machen dann auch die vielen praktischen Übungen aus, deren Höhepunkt zweifellos die Gerichtsreportage darstellt. In ihrem Vortrag sprach Merle Hilbk, freie Journalistin aus Hamburg, noch davon, die „Königsdisziplin“ solle bei der Leserin und dem Leser „Kino im Kopf“ erzeugen. Nun müssen die Teilnehmerinnen und Teilnehmer der Zusatzausbildung nach einer kurzen Einführung am frühen Morgen durch den Pressesprecher des Landgerichts schnell entscheiden, welche Gerichtsverhandlung sie besuchen wollen. Karin Völker von den Westfälischen Nachrichten erwartet den fertigen Text mittags, was den Großteil der Teilnehmerinnen und Teilnehmer zum ersten Mal den Druck eines Redaktionsschlusses spüren lässt. Schnell wird klar, wie schwer es ist, die vermeint- lich so einfachen Ratschläge der Referentinnen und Referenten umzusetzen: Aktive Sprache statt Passivkonstruktionen, kurze Sätze und klarer Satzbau statt Schachtelsätze - und natürlich: fokussieren. Da ist es kaum mehr erstaunlich, dass der Student des ersten Semesters einen der anschaulichsten Texte verfasst hat.

Die rhetorische Übung von Prof. Dr. Hoeren direkt im Anschluss stellt eine besondere Herausforderung dar. Vier Themen, teils mit aktuellem Bezug, werden verteilt. Sie sollen nach 30 Minuten Vorbereitung in eine fünfminütige Rede münden. Im Rahmen der gemeinsamen Kritik erläutert der Medienrechtler, der selbst im Rahmen seiner Priesterausbildung umfassend in der Redekunst geschult wurde, den heute immer noch gültigen Aufbau einer solchen Rede nach Cicero. Besonders Frauen empfiehlt Prof. Dr. Hoeren, auf einen ruhigen Stand zu achten, kontrollierte Gesten zu verwenden und eventuell ein Stimmtraining in Anspruch zu nehmen. „Wenn ich es Ihnen verbieten könnte, würde ich Ihnen Ihre Schüchternheit verbieten“, mahnt der Professor halb im Scherz. Man könne aber auch mit den eigenen Schwächen spielen.

Die Einblicke in den journalistischen Arbeitsalltag sind teilweise desillusionierend. Die Offenheit, mit der die Referentinnen und Referenten die Schwierigkeiten ihres Berufs schildern, ist aber auch eine besondere Bereicherung, niemals geht es um Schönfärberei. Echte Begeisterung ist bei Karin Völker von den Westfälischen Nachrichten dennoch zu spüren, wenn sie sagt, am Journalismus reize sie, dass sie so Leuten helfen könnte. Der für das ZDF tätige Fernsehjournalist Friedrich Kurz hebt die „Kombination aus Thrill, sozialer und politischer Verantwortung “ hervor.

Alle Referent/inn/en sind sich einig, dass die Verbindung von Journalismus und Juristerei viele Chancen bietet. Im Journalismus gebe es wenige Juristinnen und Juristen und so bestünde eine gewisse Nachfrage. Auch wenn die wenigsten Volljurist/ inn/en sind, empfehlen alle mit Nachdruck, das zweite Examen abzulegen. Dies würde eine größere Unabhängigkeit bedeuten.

Gefragt nach der wichtigsten Eigenschaft in seinem Job antwortet der Pressesprecher einer Großkanzlei: „Die Kernkompetenz ist dickes Fell.“ Er rät den Teilnehmerinnen und Teilnehmern: „Build your network before you need it.“

Wer sich für Journalismus interessiert, sollte sich bereits während des Grundstudiums bewerben. Für den Einstieg sind Praxiserfahrungen besonders wichtig. Dann kann man sich schon früh bei der Lokalzeitung oder auf universitärer Ebene, etwa bei einer studentischen Fachzeitschrift, dem Uni-Radio oder Uni-TV, einbringen. Der Rechtsanwalt und Online-Journalist Dr. Noogie C. Kaufmann riet aber auch zu Blogs.

Andreas Janning vom WDR schloss seinen Vortrag zuversichtlich: „Wer zu denken gelernt hat wie ein Jurist, ohne das Schreiben verlernt zu haben, wird ein guter Journalist.“ 